Published in European Journal of Operational Research, vol.

64, no. 2, pp. 278-285, which should be cited to refer to

this work.

DOI : $10.1016 / 0377-2217(93) 90182-\mathrm{M}$

\title{
BENCHMARKS \\ FOR \\ BASIC SCHEDULING PROBLEMS
}

\author{
E. TAILLARD \\ ORWP89/21 Dec. 1989
}

\begin{abstract}
In this paper, we propose 260 scheduling problems whose size is greater than that of the rare examples published. Such sizes correspond to real dimensions of industrial problems.

The types of problems that we propose are : the permutation flow shop, the job shop and the open shop scheduling problems.

We restrict us to basic problems : the processing times are fixed, there are neither set-up times nor due dates nor release dates, etc. Then, the objective is the minimization of the makespan.
\end{abstract}

Keywords : Combinatorial optimization, Scheduling, Benchmarks.

\section{Introduction}

The types of problems discussed in this paper (permutation flow shop, job shop and open shop scheduling problems) have been widely studied in the literature using exact or heuristic methods, but a common comparison base is missing. We hope that this paper will fill a gap in this domain.

The three-field nomenclature described in Lawler et al. [5] names these problems $\mathrm{F}|| \mathrm{C}_{\max }, \mathrm{J}|| \mathrm{C}_{\max }$ and $\mathrm{O}|| \mathrm{C}_{\max }$ respectively. They certainly belong to the most studied ones among the scheduling problems. Let us describe them briefly.

There are $\mathrm{n}$ jobs that have to be performed on $\mathrm{m}$ unrelated machines ; in our case, every job consists of $\mathrm{m}$ non preemptible operations. Every operation of a job uses a different machine during a given time and may wait before being processed.

For the permutation flow shop problem, the operations of every job must be processed on machines $1 \ldots \mathrm{m}$ in this order. Moreover, the processing order of the jobs on the machines is the same for every machine. The problem consists in finding a permutation of the $\mathrm{n}$ jobs that minimizes the makespan.

In the case of the job shop problem, any processing order of the jobs on the machines is allowed. For every job, the operations must be processed in a given order on the machines, but this order may differ according to the jobs.

For the open shop problem, every operation is assigned to a given machine but the order of the operations of every job is totally free.

The aim of this paper is to present unsolved problems whose size corresponds to the one of industrial problems. These problems must be easy to generate. 


\section{Generating interesting problems}

As we do not know any exact method to solve exactly the problems we want to propose, we have used heuristic methods to get hopefully good solutions of these problems. These heuristic methods are based on taboo search techniques. Taboo search is described very generally in Glover [4] and one can find some of its practical applications to the flow shop sequencing problem in Taillard [8] and Widmer et al. [10], and to the job shop scheduling problem in Taillard [9]. Taboo search is very easy to implement and generally provides excellent results, but it requires a great amount of CPU time.

In order to propose problems that are as difficult as possible (the most interesting ones), we have generated many instances of problems that we have "solved" in a summary way with taboo search. Then, we have chosen the 10 problems that seemed to be the hardest ones and we have solved them once more, allowing our heuristic method to perform a higher number of iteration.

Obviously, the choice of the hardest problems is very subjective. We decided that a problem was interesting if the best makespan we found was far from a lower bound of the makespans and if many attempts to solve the problem (starting from various initial solutions) did not provide the same solution. Such a method enabled us to detect the simplest problems but we may not propose problems that have a local optimum with a large attraction basin.

\section{The problems}

The problems we propose are randomly generated with a good random number generator proposed in Bratley [1]. We recall its implementation so that this paper is self contained.

A problem will be entirely defined by the initial value of the seed of the random generator and by the way of generating it.

For every type of problem, we give a simple manner of computing a lower bound of the makespan ; in particular, this permits to verify the generation of the problem.

For every size of problem, we give the total number of instances we have generated (summary resolution), the maximum number of iteration of taboo search that were done (long resolution) and the proportion of problems that were solved up to the lower bound, that is to say optimally. For every type and every size of problem, we give 10 instances.

For each instance, we give the initial value of the random generator seed, the best value of the makespan we have found (i. e. an upper bound of the optimal makespan) and a lower bound of all the makespans.

\section{The random number generator}

Let us recall the implementation of the linear congruential generator we have used which is based on the recursive formula $X_{i+1}=\left(\begin{array}{ll}16 & 807 X_{i}\end{array}\right) \bmod \left(2^{31}-1\right)$. This implementation uses only 32-bit integers and provides a uniformly distributed sequence of numbers between 0 and 1 (not contained) : 
0) Initial seed and $X_{0}\left(0<X_{0}<2^{31}-1\right)$

constants : $\quad a=16807, b=127773, c=2836, m=2^{31}-1$

1) Modification of $\mathrm{k}:=\left\lfloor\mathrm{X}_{\mathrm{i}} / \mathrm{b}\right\rfloor$

the seed : $\quad X_{i+1}:=a\left(X_{i} \bmod b\right)-k c$

If $X_{i+1}<0$ then let $X_{i+1}:=X_{i+1}+m$

2) New value of

the seed : $\quad X_{i+1}$

Current value of

the generator : $\quad \mathrm{X}_{\mathrm{i}+1} / \mathrm{m}$

Below, we shall denote by $\mathrm{U}(0,1)$ the pseudorandom number that this generator provides. We have $0<\mathrm{U}(0,1)<1$ for every generated number.

We shall denote by $\mathrm{U}[\mathrm{a}, \mathrm{b}]$ (with $\mathrm{a}<\mathrm{b}$, $\mathrm{a}$ and $\mathrm{b}$ integers) the integer number $\lfloor a+U(0,1) \cdot(b-a+1)\rfloor$. For every random integer generated, we have $a \leq U[a, b] \leq b$ and every integer between a and $b$ has the "same" probability of being chosen. In order to implement the integer random procedure only with 32-bit integers, the problems have been chosen in such a way that one never has to deal with a seed $\mathrm{X}$ such that :

$$
\left\lfloor\mathrm{a}+\frac{X \cdot(b-a+1)}{m}\right\rfloor \neq\left\lfloor\mathrm{a}+\frac{X}{\lfloor m /(b-a+1)\rfloor}\right\rfloor
$$

\section{Flow shop problems}

There are in the literature some problems of this type ; let us quote for example eight small and simple problems proposed in Carlier [2] and solved exactly in this reference.

The flow shop problems are characterized by the processing times $\mathrm{d}_{\mathrm{ij}}$ of job $j$ on machine $i(1 \leq \mathrm{i} \leq \mathrm{m}, 1 \leq \mathrm{j} \leq \mathrm{n})$. We have generated the values of $\mathrm{d}_{\mathrm{ij}}$ by the following way :

$$
\begin{aligned}
& \text { For } \mathrm{i}=1 \text { to } \mathrm{m} \\
& \qquad \begin{array}{l}
\text { For } \mathrm{j}=1 \text { to } \mathrm{n} \\
\qquad \mathrm{d}_{\mathrm{ij}}:=\mathrm{U}[1,99]
\end{array}
\end{aligned}
$$

We propose problems with 5, 10 and 20 machines and from 20 to 500 jobs. We compute the lower bound of the makespan as presented below.

Let $b_{i}$ be the minimum amount of time before machine $i$ starts to work and $a_{i}$ be the minimum amount of time that it remains inactive after its work up to the end of the operations, and let $\mathrm{T}_{\mathrm{i}}$ be its total processing time. We have :

$$
\begin{aligned}
& \mathrm{b}_{\mathrm{i}}=\min _{\mathrm{j}}\left(\sum_{\left.\mathrm{k}_{\mathrm{k}=1}, \mathrm{~d}_{\mathrm{kj}}\right)}^{\mathrm{i}-1} \mathrm{~m}_{\mathrm{j}} \mathrm{a}_{\mathrm{i}}=\min _{\mathrm{k}=\mathrm{i}+1}, \mathrm{~d}_{\mathrm{kj}}\right) \\
& \mathrm{T}_{\mathrm{i}}=\sum_{\mathrm{j}=1}, \mathrm{~d}_{\mathrm{ij}}
\end{aligned}
$$

Clearly, the optimal makespan $\mathrm{C}^{*}{ }_{\text {max }}$ is greater than or equal to 
$\mathrm{LB}=\max ,{ }_{i}\left(b_{i}+T_{i}+a_{i}\right) \leq C^{*}{ }^{*}$ max

This lower bound is easy to compute and we conjecture that :

$\lim _{n / m \rightarrow \infty} \operatorname{Prob}\left(\mathrm{C}^{*},{ }_{\max }=\mathrm{LB}\right)=1$

For every size of problem we give the following information (Table 1) :

Nb jobs : $\quad$ The number of jobs.

Nb machines : The number of machines.

$N b$ instances : The total number of problems generated.

$L B$ reached: The proportion of problems for which we found a solution for which the makespan was equal to the lower bound (or equal to the lower bound augmented by $2 \%$ for the 500 -job 20-machine problems).

Nb iterations: The maximum number of iterations performed by taboo search (long resolution).

Nb resolutions: The number of attempts to solve the problem from various initial solutions (long resolution).

\begin{tabular}{|r|r|r|r|r|r|}
\hline \multicolumn{1}{c|}{$\mathrm{Nb}$} & $\mathrm{Nb}$ & $\mathrm{Nb}$ & $\mathrm{LB}$ & $\mathrm{Nb}$ & $\mathrm{Nb}$ \\
\hline 20 & 5 & 100 & $35 \%$ & $10^{4}$ & 3 \\
\hline 20 & 10 & 100 & $1 \%$ & $10^{4}$ & 3 \\
\hline 20 & 20 & 100 & $0 \%$ & $2 \cdot 10^{4}$ & 3 \\
\hline 50 & 5 & 70 & $41 \%$ & $5 \cdot 10^{3}$ & 3 \\
\hline 50 & 10 & 70 & $3 \%$ & $10^{4}$ & 3 \\
\hline 50 & 20 & 70 & $0 \%$ & $5 \cdot 10^{4}$ & 3 \\
\hline 100 & 5 & 10000 & $54 \%$ & $2 \cdot 10^{3}$ & 4 \\
\hline 100 & 10 & 50 & $6 \%$ & $2 \cdot 10^{4}$ & 3 \\
\hline 100 & 20 & 50 & $0 \%$ & $10^{4}$ & 3 \\
\hline 200 & 10 & 300 & $28 \%$ & $2 \cdot 10^{3}$ & 3 \\
\hline 200 & 20 & 25 & $0 \%$ & $2 \cdot 10^{3}$ & 3 \\
\hline 500 & 20 & 100 & $14 \%$ & $10^{3}$ & 3 \\
\hline
\end{tabular}

* The value reached for this size was less than or equal to 1.02 times the lower bound.

Table 1. Flow shop problems.

Then we give ten instances for every size of problem with the following information (Table 2) :

Time seed : The initial value of the random generator's seed.

$U B$ :

An upper bound of the optimal makespan (the best value we got).

$L B$ : $\quad$ A lower bound of the makespans.

As the aim is to give an upper bound as good as possible but not a fast solving method, the computation time does not have much importance. However, let us mention 
that an iteration of taboo search needs about $4 \cdot 10^{-6} \cdot \mathrm{n}^{2} \cdot \mathrm{m}$ seconds on a "Silicon Graphics" personal workstation (10 Mips).

\begin{tabular}{|r|r|r|}
\hline \multicolumn{2}{|l|}{$\begin{array}{l}20 \text { jobs, } \\
5 \text { machines }\end{array}$} & \multicolumn{2}{c}{ Flow shop } \\
\hline Time seed & UB & LB \\
\hline 873654221 & 1278 & 1232 \\
\hline 379008056 & 1359 & 1290 \\
\hline 1866992158 & 1081 & 1073 \\
\hline 216771124 & 1293 & 1268 \\
\hline 495070989 & 1236 & 1198 \\
\hline 402959317 & 1195 & 1180 \\
\hline 1369363414 & 1239 & 1226 \\
\hline 2021925980 & 1206 & 1170 \\
\hline 573109518 & 1230 & 1206 \\
\hline 88325120 & 1108 & 1082 \\
\hline
\end{tabular}

\begin{tabular}{|r|r|l|}
\hline \multicolumn{2}{l}{$\begin{array}{l}\text { 20 jobs, } \\
10 \text { machines }\end{array}$} & \multicolumn{2}{c}{ Flow shop } & \\
\hline Time seed & UB & LB \\
\hline 587595453 & 1582 & 1448 \\
\hline 1401007982 & 1659 & 1479 \\
\hline 873136276 & 1496 & 1407 \\
\hline 268827376 & 1378 & 1308 \\
\hline 1634173168 & 1419 & 1325 \\
\hline 691823909 & 1397 & 1290 \\
\hline 73807235 & 1484 & 1388 \\
\hline 1273398721 & 1538 & 1363 \\
\hline 2065119309 & 1593 & 1472 \\
\hline 1672900551 & 1591 & 1356 \\
\hline
\end{tabular}

\begin{tabular}{|c|c|l|}
\hline \multicolumn{1}{l}{$\begin{array}{l}\text { 20 jobs, } \\
20 \text { machines }\end{array}$} & \multicolumn{2}{c}{ Flow shop } \\
\hline Time seed & UB & LB \\
\hline 479340445 & 2297 & 1911 \\
\hline 268827376 & 2100 & 1711 \\
\hline 1958948863 & 2326 & 1844 \\
\hline 918272953 & 2223 & 1810 \\
\hline 555010963 & 2291 & 1899 \\
\hline 2010851491 & 2226 & 1875 \\
\hline 1519833303 & 2273 & 1875 \\
\hline 1748670931 & 2200 & 1880 \\
\hline 1923497586 & 2237 & 1840 \\
\hline 1829909967 & 2178 & 1900 \\
\hline
\end{tabular}




\begin{tabular}{|r|r|l|}
\hline \multicolumn{1}{|l|}{$\begin{array}{l}\text { 50 jobs, } \\
\text { 5 machines }\end{array}$} & \multicolumn{1}{c|}{ Flow shop } & \\
\hline Time seed & UB & LB \\
\hline 1328042058 & 2724 & 2712 \\
\hline 200382020 & 2836 & 2808 \\
\hline 496319842 & 2621 & 2596 \\
\hline 1203030903 & 2751 & 2740 \\
\hline 1730708564 & 2863 & 2837 \\
\hline 450926852 & 2829 & 2793 \\
\hline 1303135678 & 2725 & 2689 \\
\hline 1273398721 & 2683 & 2667 \\
\hline 587288402 & 2554 & 2527 \\
\hline 248421594 & 2782 & 2776 \\
\hline
\end{tabular}

\begin{tabular}{|c|c|c|}
\hline $\begin{array}{l}50 \text { jobs, } \\
10 \text { machines }\end{array}$ & Flow shop & \\
\hline Time seed & UB & LB \\
\hline 1958948863 & 3037 & 2907 \\
\hline 575633267 & 2911 & 2821 \\
\hline 655816003 & 2873 & 2801 \\
\hline 1977864101 & 3067 & 2968 \\
\hline 93805469 & 3025 & 2908 \\
\hline 1803345551 & 3021 & 2941 \\
\hline 49612559 & 3124 & 3062 \\
\hline 1899802599 & 3048 & 2959 \\
\hline 2013025619 & 2913 & 2795 \\
\hline 578962478 & 3114 & 3046 \\
\hline
\end{tabular}

\begin{tabular}{|c|c|c|}
\hline \multicolumn{1}{|l|}{$\begin{array}{l}\text { 50 jobs, } \\
\text { 20 machines }\end{array}$} & \multicolumn{1}{c}{ Flow shop } & \\
\hline Time seed & UB & LB \\
\hline 1539989115 & 3886 & 3480 \\
\hline 691823909 & 3733 & 3424 \\
\hline 655816003 & 3689 & 3351 \\
\hline 1315102446 & 3755 & 3336 \\
\hline 1949668355 & 3655 & 3313 \\
\hline 1923497586 & 3719 & 3460 \\
\hline 1805594913 & 3730 & 3427 \\
\hline 1861070898 & 3744 & 3383 \\
\hline 715643788 & 3790 & 3457 \\
\hline 464843328 & 3791 & 3438 \\
\hline
\end{tabular}




\begin{tabular}{|c|c|c|}
\hline \multicolumn{1}{l}{$\begin{array}{l}\text { 100 jobs, } \\
\text { 5 machines }\end{array}$} & \multicolumn{1}{c}{ Flow shop } & \\
\hline Time seed & UB & LB \\
\hline 896678084 & 5493 & 5437 \\
\hline 1179439976 & 5274 & 5208 \\
\hline 1122278347 & 5175 & 5130 \\
\hline 416756875 & 5018 & 4963 \\
\hline 267829958 & 5250 & 5195 \\
\hline 1835213917 & 5135 & 5063 \\
\hline 1328833962 & 5247 & 5198 \\
\hline 1418570761 & 5106 & 5038 \\
\hline 161033112 & 5454 & 5385 \\
\hline 304212574 & 5328 & 5272 \\
\hline
\end{tabular}

\begin{tabular}{|c|c|c|}
\hline $\begin{array}{l}100 \text { jobs, } \\
10 \text { machines }\end{array}$ & Flow sho & \\
\hline Time seed & UB & $\mathrm{LB}$ \\
\hline 1539989115 & 5776 & 5759 \\
\hline 655816003 & 5362 & 5345 \\
\hline 960914243 & 5679 & 5623 \\
\hline 1915696806 & 5820 & 5732 \\
\hline 2013025619 & 5491 & 5431 \\
\hline 1168140026 & 5308 & 5246 \\
\hline 1923497586 & 5602 & 5523 \\
\hline 167698528 & 5640 & 5556 \\
\hline 1528387973 & 5891 & 5779 \\
\hline 993794175 & 5860 & 5830 \\
\hline
\end{tabular}

\begin{tabular}{|r|r|l|}
\hline \multicolumn{1}{l}{$\begin{array}{l}\text { 100 jobs, } \\
\text { 20 machines }\end{array}$} & \multicolumn{2}{c}{ Flow shop } \\
\hline Time seed & UB & LB \\
\hline 450926852 & 6345 & 5851 \\
\hline 1462772409 & 6323 & 6099 \\
\hline 1021685265 & 6385 & 6099 \\
\hline 83696007 & 6331 & 6072 \\
\hline 508154254 & 6405 & 6009 \\
\hline 1861070898 & 6487 & 6144 \\
\hline 26482542 & 6393 & 5991 \\
\hline 444956424 & 6514 & 6084 \\
\hline 2115448041 & 6386 & 5979 \\
\hline 118254244 & 6544 & 6298 \\
\hline
\end{tabular}




\begin{tabular}{|c|c|c|}
\hline \multicolumn{2}{|l|}{$\begin{array}{l}\text { 200 jobs, } \\
10 \text { machines }\end{array}$} & \multicolumn{2}{c}{ Flow shop } & \\
\hline Time seed & UB & LB \\
\hline 471503978 & 10927 & 10816 \\
\hline 1215892992 & 10570 & 10422 \\
\hline 135346136 & 11004 & 10886 \\
\hline 1602504050 & 10936 & 10794 \\
\hline 160037322 & 10550 & 10437 \\
\hline 551454346 & 10378 & 10255 \\
\hline 519485142 & 10885 & 10761 \\
\hline 383947510 & 10808 & 10663 \\
\hline 1968171878 & 10473 & 10348 \\
\hline 540872513 & 10727 & 10616 \\
\hline
\end{tabular}

\begin{tabular}{|c|c|c|}
\hline \multicolumn{2}{|l|}{$\begin{array}{l}\text { 200 jobs, } \\
\text { 20 machines }\end{array}$} & \multicolumn{1}{c}{ Flow shop } \\
\hline Time seed & UB & LB \\
\hline 2013025619 & 11441 & 10979 \\
\hline 475051709 & 11549 & 10947 \\
\hline 914834335 & 11537 & 11150 \\
\hline 810642687 & 11580 & 11127 \\
\hline 1019331795 & 11484 & 11132 \\
\hline 2056065863 & 11416 & 11085 \\
\hline 1342855162 & 11659 & 11194 \\
\hline 1325809384 & 11587 & 11126 \\
\hline 1988803007 & 11498 & 10965 \\
\hline 765656702 & 11569 & 11122 \\
\hline
\end{tabular}

\begin{tabular}{|r|c|c|}
\hline \multicolumn{2}{|l|}{$\begin{array}{l}\text { 500 jobs, } \\
\text { 20 machines }\end{array}$} & \multicolumn{1}{c}{ Flow shop } \\
\hline Time seed & UB & LB \\
\hline 1368624604 & 26699 & 25922 \\
\hline 450181436 & 27303 & 26353 \\
\hline 1927888393 & 26928 & 26320 \\
\hline 1759567256 & 27009 & 26424 \\
\hline 606425239 & 26771 & 26181 \\
\hline 19268348 & 26959 & 26401 \\
\hline 1298201670 & 26870 & 26300 \\
\hline 2041736264 & 27104 & 26429 \\
\hline 379756761 & 26586 & 25891 \\
\hline 28837162 & 26910 & 26315 \\
\hline
\end{tabular}

Table 2. Instances of flow shop problems. 


\section{Job shop problems}

In the literature, we may find instances of small problems in Lawrence [6] and Muth et al. [7] ; most of the optimal values of these problems are given in Carlier et al. [3]. We can consider that problems up to ten machines may be solved satisfactorily with existing methods. This is why we propose problems with 15 and 20 machines and from 15 to 100 jobs.

The processing time $\mathrm{d}_{\mathrm{ij}}$ of the jth operation of job $i,(1 \leq \mathrm{i} \leq \mathrm{n}, 1 \leq \mathrm{j} \leq \mathrm{m})$ is obtained as follows :

$$
\begin{aligned}
& \text { For } \mathrm{i}=1 \text { to } \mathrm{n} \\
& \qquad \begin{array}{l}
\text { For } \mathrm{j}=1 \text { to } \mathrm{m} \\
\mathrm{d}_{\mathrm{ij}}:=\mathrm{U}[1,99]
\end{array}
\end{aligned}
$$

The machine $\mathrm{M}_{\mathrm{ij}}$ on which the jth operation of job $i$ has to be performed is given by the following procedure :

0) $\quad \mathrm{M}_{\mathrm{ij}}:=\mathrm{j}(1 \leq \mathrm{i} \leq \mathrm{n}, 1 \leq \mathrm{j} \leq \mathrm{m})$

1) For $\mathrm{i}=1$ to $\mathrm{n}$

$$
\begin{aligned}
\text { For } \mathrm{j}= & 1 \text { to } \mathrm{m} \\
& \text { Swap } \mathrm{M}_{\mathrm{ij}} \text { and } \mathrm{M}_{\mathrm{iU}[\mathrm{j}, \mathrm{m}]}
\end{aligned}
$$

Let us note the use of another initial seed for the choice of the machines : Machine seed.

An instance of a small open shop problem, obtained with the same procedures, is given extensively in Table 7 .

The lower bound of the makespans corresponds to the maximum amount of time that a job or a machine requires, i.e. :

$$
\mathrm{LB}=\max \left\{\max _{\mathrm{j}}\left(\sum, \mathrm{d}_{\mathrm{ij}}\right), \max _{\mathrm{i}}\left(\sum_{j, k \mid M_{k i}=i} \mathrm{~d}_{\mathrm{kj}}\right)\right\}
$$

We conjecture again that this bound is tight if $\mathrm{n} / \mathrm{m} \rightarrow \infty$, because we have always found an optimal schedule if $\mathrm{n} / \mathrm{m} \geq 6$, considering more than 2000 problems whose size was varying from 20 jobs, 2 machines to 150 jobs, 15 machines, passing by 500 jobs, 4 machines.

The time needed to perform one iteration of taboo search is about $20 \cdot 10^{-6} \cdot \mathrm{n} \cdot \mathrm{m}$ seconds on the same computer as for the flow shop problems.

Tables 3 and 4 are analogous to Tables 1 and 2, but, for job shop problems, we give in addition Machine seed in Table 4. 


\begin{tabular}{|c|c|c|c|c|c|}
\hline $\begin{array}{c}\mathrm{Nb} \\
\text { jobs }\end{array}$ & $\begin{array}{c}\mathrm{Nb} \\
\text { machines }\end{array}$ & $\begin{array}{c}\mathrm{Nb} \\
\text { instance } \\
\mathrm{s}\end{array}$ & $\begin{array}{c}\mathrm{LB} \\
\text { reached }\end{array}$ & $\begin{array}{c}\mathrm{Nb} \\
\text { iterations }\end{array}$ & $\begin{array}{c}\mathrm{Nb} \\
\text { resolutions }\end{array}$ \\
\hline 15 & 15 & 50 & $0 \%$ & $5 \cdot 10^{5}$ & 4 \\
\hline 20 & 15 & 50 & $4 \%$ & $10^{6}$ & 3 \\
\hline 20 & 20 & 50 & $0 \%$ & $10^{7}$ & 4 \\
\hline 30 & 15 & 50 & $18 \%$ & $5 \cdot 10^{5}$ & 4 \\
\hline 30 & 20 & 50 & $0 \%$ & $2 \cdot 10^{6}$ & 4 \\
\hline 50 & 15 & 100 & $65 \%$ & $2 \cdot 10^{5}$ & 4 \\
\hline 50 & 20 & 26 & $15 \%$ & $5 \cdot 10^{5}$ & 4 \\
\hline 100 & 20 & 100 & $89 \%$ & $3 \cdot 10^{5}$ & 3 \\
\hline
\end{tabular}

Table 3. Job shop problems.

\begin{tabular}{|r|r|r|r|}
\hline \multicolumn{1}{c}{$\begin{array}{l}\text { 15 jobs, } \\
15 \text { machines }\end{array}$} & \multicolumn{2}{|c|}{ Job shop } & \\
\hline Time seed & Machine seed & UB & LB \\
\hline 840612802 & 398197754 & 1247 & 977 \\
\hline 1314640371 & 386720536 & 1263 & 942 \\
\hline 1227221349 & 316176388 & 1233 & 921 \\
\hline 342269428 & 1806358582 & 1181 & 911 \\
\hline 1603221416 & 1501949241 & 1236 & 940 \\
\hline 1357584978 & 1734077082 & 1247 & 889 \\
\hline 44531661 & 1374316395 & 1235 & 935 \\
\hline 302545136 & 2092186050 & 1221 & 963 \\
\hline 1153780144 & 1393392374 & 1289 & 982 \\
\hline 73896786 & 1544979948 & 1270 & 911 \\
\hline
\end{tabular}

\begin{tabular}{|c|c|c|c|}
\hline $\begin{array}{l}20 \text { jobs, } \\
15 \text { machines }\end{array}$ & & Job sh & \\
\hline Time seed & Machine seed & $\mathrm{UB}$ & $\mathrm{LB}$ \\
\hline 533484900 & 317419073 & 1376 & 1139 \\
\hline 1894307698 & 1474268163 & 1381 & 1251 \\
\hline 874340513 & 509669280 & 1368 & 1178 \\
\hline 1124986343 & 1209573668 & 1356 & 1130 \\
\hline 1463788335 & 529048107 & 1375 & 1148 \\
\hline 1056908795 & 25321885 & 1385 & 1181 \\
\hline 195672285 & 1717580117 & 1495 & 1257 \\
\hline 961965583 & 1353003786 & 1432 & 1153 \\
\hline 1610169733 & 1734469503 & 1378 & 1202 \\
\hline 532794656 & 998486810 & 1383 & 1186 \\
\hline
\end{tabular}




\begin{tabular}{|c|c|c|c|}
\hline $\begin{array}{l}20 \text { jobs, } \\
20 \text { machines }\end{array}$ & & Job sh & \\
\hline Time seed & Machine seed & UB & LB \\
\hline 1035939303 & 773961798 & 1663 & 1217 \\
\hline 5997802 & 1872541150 & 1626 & 1240 \\
\hline 1357503601 & 722225039 & 1574 & 1185 \\
\hline 806159563 & 1166962073 & 1665 & 1271 \\
\hline 1902815253 & 1879990068 & 1598 & 1256 \\
\hline 1503184031 & 1850351876 & 1679 & 1207 \\
\hline 1032645967 & 99711329 & 1704 & 1331 \\
\hline 229894219 & 1158117804 & 1633 & 1269 \\
\hline 823349822 & 108033225 & 1635 & 1267 \\
\hline 1297900341 & 489486403 & 1616 & 1212 \\
\hline
\end{tabular}

\begin{tabular}{|r|r|r|r|}
\hline \multicolumn{1}{|c|}{$\begin{array}{l}\text { 10 jobs, } \\
10 \text { machines }\end{array}$} & \multicolumn{2}{|c|}{ Open shop } & \\
\hline Time seed & Machine seed & UB & LB \\
\hline 1344106948 & 1868311537 & 652 & 637 \\
\hline 425990073 & 1111853152 & 596 & 588 \\
\hline 666128954 & 1750328066 & 617 & 598 \\
\hline 442723456 & 1369177184 & 581 & 577 \\
\hline 2033800800 & 1344077538 & 657 & 640 \\
\hline 964467313 & 1735817385 & 545 & 538 \\
\hline 1004528509 & 967002400 & 623 & 616 \\
\hline 1667495107 & 818777384 & 606 & 595 \\
\hline 1806968543 & 1561913259 & 606 & 595 \\
\hline 938376228 & 344628625 & 604 & 596 \\
\hline
\end{tabular}

\begin{tabular}{|c|c|c|c|}
\hline $\begin{array}{l}30 \text { jobs, } \\
15 \text { machines }\end{array}$ & & Job sh & \\
\hline Time seed & Machine seed & UB & LB \\
\hline 98640593 & 1981283465 & 1770 & 1764 \\
\hline 1839268120 & 248890888 & 1853 & 1774 \\
\hline 573875290 & 2081512253 & 1864 & 1729 \\
\hline 1670898570 & 788294565 & 1852 & 1828 \\
\hline 1118914567 & 1074349202 & 2015 & 1729 \\
\hline 178750207 & 294279708 & 1844 & 1777 \\
\hline 1549372605 & 596993084 & 1823 & 1771 \\
\hline 798174738 & 151685779 & 1714 & 1673 \\
\hline 553410952 & 1329272528 & 1824 & 1641 \\
\hline 1661531649 & 1173386294 & 1723 & 1602 \\
\hline
\end{tabular}




\begin{tabular}{|r|r|r|r|}
\hline \multicolumn{1}{l}{$\begin{array}{l}\text { 30 jobs, } \\
\text { 20 machines }\end{array}$} & \multicolumn{2}{c}{ Job shop } & \\
\hline Time seed & Machine seed & UB & LB \\
\hline 1841414609 & 1357882888 & 2064 & 1830 \\
\hline 2116959593 & 1546338557 & 1983 & 1761 \\
\hline 796392706 & 1230864158 & 1905 & 1694 \\
\hline 532496463 & 254174057 & 2031 & 1787 \\
\hline 2020525633 & 978943053 & 2038 & 1731 \\
\hline 524444252 & 185526083 & 2057 & 1856 \\
\hline 1569394691 & 487269855 & 1950 & 1690 \\
\hline 1460267840 & 1631446539 & 2014 & 1744 \\
\hline 198324822 & 1937476577 & 2013 & 1758 \\
\hline 38071822 & 1541985579 & 1973 & 1674 \\
\hline
\end{tabular}

\begin{tabular}{|r|r|r|l|}
\hline \multicolumn{1}{|l}{$\begin{array}{l}\text { 50 jobs, } \\
15 \text { machines }\end{array}$} & \multicolumn{2}{c}{ Job shop } & \\
\hline Time seed & Machine seed & UB & LB \\
\hline 17271 & 718939 & 2791 & 2760 \\
\hline 660481279 & 449650254 & 2800 & 2756 \\
\hline 352229765 & 949737911 & 2768 & 2717 \\
\hline 1197518780 & 166840558 & 2845 & 2797 \\
\hline 1376020303 & 483922052 & 2757 & 2679 \\
\hline 2106639239 & 955932362 & 2833 & 2781 \\
\hline 1765352082 & 1209982549 & 2977 & 2943 \\
\hline 1105092880 & 1349003108 & 2928 & 2885 \\
\hline 907248070 & 919544535 & 2722 & 2655 \\
\hline 2011630757 & 1845447001 & 2777 & 2723 \\
\hline
\end{tabular}

\begin{tabular}{|c|c|c|c|}
\hline $\begin{array}{l}50 \text { jobs, } \\
20 \text { machines }\end{array}$ & & Job sh & \\
\hline Time seed & Machine seed & UB & LB \\
\hline 8493988 & 2738939 & 2961 & 2868 \\
\hline 1991925010 & 709517751 & 3013 & 2848 \\
\hline 342093237 & 786960785 & 2859 & 2755 \\
\hline 1634043183 & 973178279 & 2790 & 2691 \\
\hline 341706507 & 286513148 & 2813 & 2725 \\
\hline 320167954 & 1411193018 & 2921 & 2845 \\
\hline 1089696753 & 298068750 & 2907 & 2812 \\
\hline 433032965 & 1589656152 & 2840 & 2764 \\
\hline 615974477 & 331205412 & 3129 & 3063 \\
\hline 236150141 & 592292984 & 3173 & 2995 \\
\hline
\end{tabular}




\begin{tabular}{|r|r|r|l|}
\hline \multicolumn{1}{|l}{$\begin{array}{l}\text { 100 jobs, } \\
20 \text { machines }\end{array}$} & \multicolumn{2}{c|}{ Job shop } & \\
\hline Time seed & Machine seed & UB & LB \\
\hline 302034063 & 1203569070 & 5582 & 5464 \\
\hline 1437643198 & 1692025209 & 5221 & 5181 \\
\hline 1792475497 & 1039908559 & 5671 & 5552 \\
\hline 1647273132 & 1012841433 & 5345 & 5339 \\
\hline 696480901 & 1689682358 & 5573 & 5392 \\
\hline 1785569423 & 1092647459 & 5403 & 5342 \\
\hline 117806902 & 739059626 & 5450 & 5436 \\
\hline 1639154709 & 1319962509 & 5459 & 5394 \\
\hline 2007423389 & 749368241 & 5360 & 5358 \\
\hline 682761130 & 262763021 & 5278 & 5183 \\
\hline
\end{tabular}

Table 4. Instances of Job shop problems.

\section{Open shop problems}

We do not know instances of such problems in the literature. This is why we give problems of small size. These problems are obtained using exactly the same procedures as those used for the job shop problems, and the lower bound remains the same too.

Because one has to choose the order of the operations of a job, one can find very often an optimal schedule, except for the problems in which the number of jobs is about the number of machines. In this case, either an optimal solution is easily reached, or the problem is harder than a job shop problems of the same size.

For problems with $\mathrm{n} \gg \mathrm{m}$, we have observed empirically that the mean complexity of taboo search applied to open shop problems - $\mathrm{O}\left(\mathrm{n}^{2.37} \cdot \mathrm{m}^{3.69}\right)$ - is lower than the complexity of taboo search applied to job shop problems $-\mathrm{O}\left(\mathrm{n}^{2.50} \cdot \mathrm{m}^{3.81}\right)$.

In table 7, we describe extensively the first 4-job 4-machine problem we propose, i.e. the processing times $\mathrm{d}_{\mathrm{ij}}$ of the operation $j$ of job $i$ and its associated machine $\mathrm{M}_{\mathrm{ij}}$. We give a good (optimal ?) schedule of this problem in the Gantt chart of Figure 1.

The time needed by taboo search to perform one iteration is about $23 \cdot 10^{-6} \cdot \mathrm{n} \cdot \mathrm{m} \mathrm{sec}-$ onds.

Tables 5 and 6 are analogous to Tables 3 and 4.

\begin{tabular}{|c|c|c|c|c|c|}
\hline $\begin{array}{c}\mathrm{Nb} \\
\text { jobs }\end{array}$ & $\begin{array}{c}\mathrm{Nb} \\
\text { machin } \\
\text { es }\end{array}$ & $\begin{array}{c}\mathrm{Nb} \\
\text { instances }\end{array}$ & $\begin{array}{c}\mathrm{LB} \\
\text { reached }\end{array}$ & $\begin{array}{c}\mathrm{Nb} \\
\text { iterations }\end{array}$ & $\begin{array}{c}\mathrm{Nb} \\
\text { resoluti } \\
\text { ons }\end{array}$ \\
\hline 4 & 4 & $50^{\prime} 000$ & $98.5 \%$ & $10^{5}$ & 5 \\
\hline 5 & 5 & $45^{\prime} 000$ & $99.7 \%$ & $5 \cdot 10^{5}$ & 4 \\
\hline 7 & 7 & $1^{\prime} 000$ & $94 \%$ & $10^{6}$ & 5 \\
\hline 10 & 10 & 300 & $89 \%$ & $2 \cdot 10^{6}$ & 5 \\
\hline 15 & 15 & 40 & $52 \%$ & $3 \cdot 10^{5}$ & 3 \\
\hline 20 & 20 & 25 & $24 \%$ & $3 \cdot 10^{6}$ & 3 \\
\hline
\end{tabular}

Table 5. Open shop problems. 


\begin{tabular}{|r|r|r|r|}
\hline \multicolumn{1}{|l}{$\begin{array}{l}\text { U jobs, } \\
4 \text { machines }\end{array}$} & \multicolumn{2}{c|}{ Open shop } & \\
\hline Time seed & Machine seed & UB & LB \\
\hline 1166510396 & 164000672 & 193 & 186 \\
\hline 1624514147 & 1076870026 & 236 & 229 \\
\hline 1116611914 & 1729673136 & 271 & 262 \\
\hline 410579806 & 1453014524 & 250 & 245 \\
\hline 1036100146 & 375655500 & 295 & 287 \\
\hline 597897640 & 322140729 & 189 & 185 \\
\hline 1268670769 & 556009645 & 201 & 197 \\
\hline 307928077 & 421384574 & 217 & 212 \\
\hline 667545295 & 485515899 & 261 & 258 \\
\hline 35780816 & 492238933 & 217 & 213 \\
\hline
\end{tabular}

\begin{tabular}{|r|r|r|r|}
\hline \multicolumn{1}{|l}{ 5 jobs, } & \multicolumn{2}{c|}{ Open shop } & \\
\hline 5 machines & & UB & LB \\
\hline Time seed & Machine seed & 300 & 295 \\
\hline 527556884 & 1343124817 & 262 & 255 \\
\hline 1046824493 & 1973406531 & 328 & 321 \\
\hline 1165033492 & 86711717 & 310 & 306 \\
\hline 476292817 & 24463110 & 329 & 321 \\
\hline 1181363416 & 606981348 & 312 & 307 \\
\hline 897739730 & 513119113 & 305 & 298 \\
\hline 577107303 & 2046387124 & 300 & 292 \\
\hline 1714191910 & 1928475945 & 353 & 349 \\
\hline 1813128617 & 2091141708 & 326 & 321 \\
\hline 808919936 & 183753764 & & \\
\hline
\end{tabular}

\begin{tabular}{|r|r|r|r|}
\hline \multicolumn{1}{|l}{$\begin{array}{c}\text { O jobs, } \\
7 \text { machines }\end{array}$} & \\
\hline Time seed & Machine seed & UB & LB \\
\hline 1840686215 & 1827454623 & 438 & 435 \\
\hline 1026771938 & 1312166461 & 449 & 443 \\
\hline 609471574 & 670843185 & 479 & 468 \\
\hline 1022295947 & 398226875 & 467 & 463 \\
\hline 1513073047 & 1250759651 & 419 & 416 \\
\hline 1612211197 & 95606345 & 460 & 451 \\
\hline 435024109 & 1118234860 & 435 & 422 \\
\hline 1760865440 & 1099909092 & 426 & 424 \\
\hline 122574075 & 10979313 & 460 & 458 \\
\hline 248031774 & 1685251301 & 400 & 398 \\
\hline
\end{tabular}




\begin{tabular}{|r|r|r|r|}
\hline \multicolumn{1}{|c}{$\begin{array}{c}\text { 15 jobs, } \\
15 \text { machines }\end{array}$} & \multicolumn{2}{c|}{ Open shop } & \\
\hline Time seed & Machine seed & UB & LB \\
\hline 1561423441 & 1787167667 & 956 & 937 \\
\hline 204120997 & 213027331 & 957 & 918 \\
\hline 801158374 & 1812110433 & 899 & 871 \\
\hline 1502847623 & 1527847153 & 946 & 934 \\
\hline 282791231 & 1855451778 & 992 & 946 \\
\hline 1130361878 & 849417380 & 959 & 933 \\
\hline 379464508 & 944419714 & 931 & 891 \\
\hline 1760142791 & 1955448160 & 916 & 893 \\
\hline 1993140927 & 179408412 & 951 & 899 \\
\hline 1678386613 & 1567160817 & 935 & 902 \\
\hline
\end{tabular}

20 jobs,

20 machines

Open shop

\begin{tabular}{|r|r|r|l|}
\hline Time seed & Machine seed & UB & LB \\
\hline 957638 & 9237185 & 1215 & 1155 \\
\hline 162587311 & 1489531109 & 1332 & 1241 \\
\hline 965299017 & 1054695706 & 1294 & 1257 \\
\hline 1158457671 & 1499999517 & 1310 & 1248 \\
\hline 1191143707 & 1530757746 & 1301 & 1256 \\
\hline 1826671743 & 901609771 & 1252 & 1204 \\
\hline 1591533998 & 1146547719 & 1352 & 1294 \\
\hline 937297777 & 92726463 & 1269 & 1169 \\
\hline 687896268 & 1731298717 & 1322 & 1289 \\
\hline 687034842 & 684013066 & 1284 & 1241 \\
\hline
\end{tabular}

Table 6. Instances of Open shop problems.

\begin{tabular}{|c|r|r|r|r|}
\cline { 2 - 5 } \multicolumn{1}{c|}{} & \multicolumn{4}{|c|}{ Operation $j$} \\
\hline Job $i$ & 1 & 2 & 3 & 4 \\
\hline 1 & 54 & 34 & 61 & 2 \\
\hline 2 & 9 & 15 & 89 & 70 \\
\hline 3 & 38 & 19 & 28 & 87 \\
\hline 4 & 95 & 34 & 7 & 29 \\
\hline
\end{tabular}

a) Processing times $\left(d_{i j}\right)$ 


\begin{tabular}{|c|c|c|c|c|}
\cline { 2 - 5 } \multicolumn{1}{c|}{} & \multicolumn{4}{|c|}{ Operation $j$} \\
\hline Job $i$ & 1 & 2 & 3 & 4 \\
\hline 1 & 3 & 1 & 4 & 2 \\
\hline 2 & 4 & 1 & 2 & 3 \\
\hline 3 & 1 & 2 & 3 & 4 \\
\hline 4 & 1 & 3 & 2 & 4 \\
\hline
\end{tabular}

b) Machines $\left(\mathbf{M}_{\mathrm{ij}}\right)$

Table 7. The first instance of the 4-job 4-machine open shop problem.

\section{Concluding remarks}

We hope that the problems that we propose will constitute a comparison base for the future resolution methods.

Everyone may send us his own results about these problems, specifying whether his solutions are proved optimal or not, in order to update the best solutions known.

\section{References}

[1] P. Bratley, B.L. Fox \& L.E. Schrage, "A guide to Simulation", Springer Verlag, New-York, 1983.

[2] J. Carlier, "Problèmes d'ordonnancement à contraintes de ressources : algorithmes et complexité", Méthodologie \& architecture des systèmes informatiques, Institut de programmation, Université P.\& M. Curie, Paris, 1984.

[3] J. Carlier \& E. Pinson, "An algorithm for solving the job-shop problem”, Management Science, Vol 35 No 2, 164-176, 1989.

[4] F. Glover, "Tabu Search - Part I", ORSA journal on computing, 1, 190-206, 1989.

[5] E.L. Lawler, J.K. Lenstra, A.H.G. Rinnooy Kan \& D.B. Shmoys, "Sequencing and scheduling", Report BS-R89xx, Centre for Mathematics and Computer Science, Amsterdam, the Netherlands, 1989.

[6] S. Lawrence, "Resource constrained project Scheduling : An Experimental Investigation of Heuristic Scheduling Techniques (supplement)", Graduate School of Industrial Administration,.Carnegie Mellon University, 1982.

[7] J.F. Muth \& G.L. Thompson, "Industrial Scheduling”, Prentice Hall, Englewood Cliffs, N.J., 225-251, 1963.

[8] E. Taillard, "Some efficient heuristic methods for the flowshop sequencing problem”, Report ORWP 88/12, DMA, Ecole Polytechnique Fédérale de Lausanne, Lausanne, Switzerland, (To appear in European Journal of Operational Research), 1988. 
[9] E. Taillard, "Parallel taboo search technique for the jobshop scheduling problem", ORWP 89/11, DMA, Ecole Polytechnique Fédérale de Lausanne, Lausanne, Switzerland, 1989.

[10] M. Widmer \& A. Hertz, "A new heuristic for the flowshop sequencing problem”, European Journal of Operational Research vol 41 (2), 186-193, 1989.

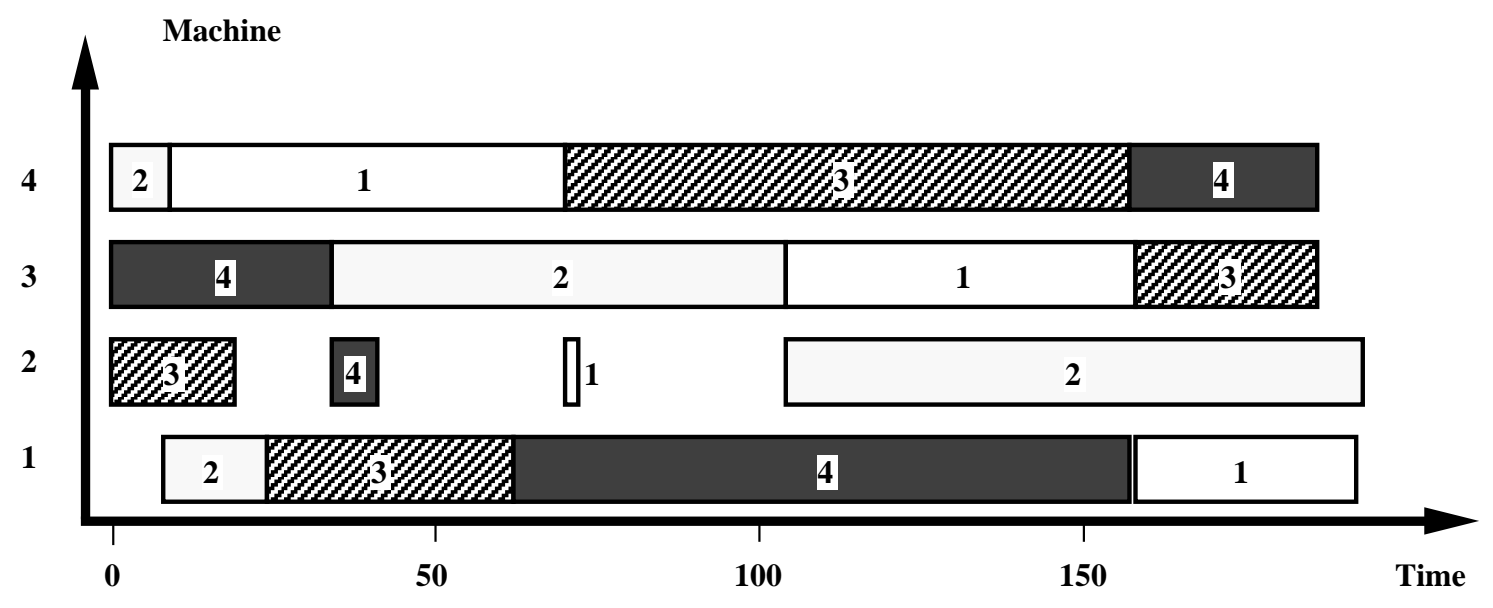

Figure 1. A good schedule for the problem of Table 7. 